\title{
Fuzzy preference modelling and some consequences for fuzzy implications and quasi-copulas
}

\author{
Dana Hliněná ${ }^{1}$ Martin Kalina ${ }^{2}$ Pavol Král ${ }^{3}$ \\ ${ }^{1}$ Dept. of Mathematics FEEC Brno Uni. of Technology \\ Technická 8, Cz-616 00 Brno, Czech Republic \\ e-mail: hlinena@feec.vutbr.cz \\ ${ }^{2}$ Slovak University of Technology in Bratislava \\ Faculty of Civil Engineering, Department of Mathematics \\ Radlinského 11, Sk-813 68 Bratislava, Slovakia \\ e-mail: kalina@math.sk \\ ${ }^{3}$ Dept. of Quantitative Methods and Information Systems \\ Faculty of Economics, Matej Bel University \\ Tajovského 10, Sk-975 90 Banská Bystrica \\ Slovakia \\ e-mail: pavol.kral@umb.sk
}

\begin{abstract}
In this paper we study a connection between quasicopulas and generator triples of fuzzy preference structures. We study also conditions under which fuzzy implications can be used to construct generator triples. As we show, an important property of fuzzy implications that enables us to construct a generator triple from a fuzzy implication, is the contrapositive symmetry.
\end{abstract}

Keywords: Contrapositive symmetry, fuzzy implication, fuzzy preference structure, generator triple, quasi-copula

\section{Introduction, basic notions and known facts}

The aim of this paper is to show how fuzzy preference structures can be constructed via commutative quasi-copulas. We show also some consequences of these constructions to fuzzy implications. To make the paper self-contained we repeat basic definitions and known results on fuzzy preference structures and fuzzy connectives that will be important for our considerations.

\subsection{Fuzzy logic and basic connectives}

Definition 1 (See e.g. [4]) A decreasing function $N:[0,1] \rightarrow[0,1]$ with $N(0)=1$ and $N(1)=0$ is called a fuzzy negation.

If $N$ is an involutive fuzzy negation, i.e. $N(N(x))=$ $x$ for all $x \in[0,1]$, we say that $N$ is a strong fuzzy negation.

Commonly used negation in applications is the standard negation $N_{s}(x)=1-x$.
Generalisations of binary conjunctions in fuzzy logic are conjunctors.

Definition 2 (see, e.g., [3]) A function Con : $[0,1]^{2} \rightarrow[0,1]$ is called a conjunctor, if it is increasing in both places and such that $\operatorname{Con}(x, 0)=$ $\operatorname{Con}(0, x)=0$ for all $x \in[0,1]$, and $\operatorname{Con}(1,1)=1$.

There are special classes of conjunctors. The following one will be important for us.

- A semi-copula (see [2]) is a conjunctor with 1 as neutral element.

- A quasi-copula (see, e.g., [9]) is a semi-copula that is 1-Lipschitz in both variables.

- A t-norm (see, e.g. [8]) is a commutative and associative conjunctor with 1 as neutral element.

T-norms serve as basic associative and commutative conjunctors.

There are three basic continuous t-norms: minimum $\left(T_{M}\right)$, product $\left(T_{P}\right)$ and the Eukasiewicz tnorm

$$
T_{L}(x, y)=\max \{0, x+y-1\} .
$$

Remark 1 The three basic t-norms are also copulas. The notation used in the theory of copulas is $M$ for minimum, $\Pi$ for the product and $W$ for the Łukasiewicz t-norm. $M$ and $W$ are lower and upper Fréchet-Hoeffding bounds, respectively. In the rest of the paper we use the notation standard in the theory of t-norms.

Let $N$ be a strong fuzzy negation. To each conjunctor Con there exists an $N$-dual connective called disjunctor (see, e.g., [3]), i.e.,

$$
\operatorname{Dis}(x, y)=N(\operatorname{Con}(N(x), N(y)) .
$$


An $N$-dual disjunctor to a t-norm $T$ is called a $t$-conorm usually is denoted by $S$. Particularly, the $N_{s}$-dual t-conorm to $T_{L}$ is denoted by $S_{L}$ and is called the Eukasiewicz t-conorm. The $N_{s}$-dual tconorm to $T_{M}$ is denoted by $S_{M}$.

Let $T$ be a continuous t-norm, $N$ be a strong fuzzy negation and $S$ be the $N$-dual t-conorm to $T$. Then we say that $(T, S, N)$ is a De Morgan triple.

In literature we can find several definitions of fuzzy implications. We adopt the one equivalent with the definition introduced by Fodor and Roubens [4].

Definition 3 ([4]) A function $I:[0,1]^{2} \rightarrow[0,1]$ is called a fuzzy implication if it satisfies the following conditions:

(I1) I is decreasing in its first variable,

(I2) I is increasing in its second variable,

(I3) $I(1,0)=0, I(0,0)=I(1,1)=1$.

There are several construction methods for fuzzy implications (see, e.g., [1]). Among them a wellknown method is given by the following

$$
I(x, y)=\operatorname{Dis}(N(x), y),
$$

where Dis is an arbitrary disjunctor and $N$ a fuzzy negation. Such implications are called (Dis, $N$ )implications. The most important for us will be the family of $(S, N)$ implications, where $S$ is a t-conorm and $N$ is a strong fuzzy negation. For $N=N_{s}$ the fuzzy implication $I(x, y)=S(1-x, y)$ will be denoted by $I_{S}$.

To each fuzzy implication $I$ we can assign a fuzzy negation that is called natural negation and denoted by $N_{I}$ :

$$
N_{I}(x)=I(x, 0)
$$

Next we list some important properties of fuzzy implications. For more information one can consult [6] or [10].

Definition 4 Let $x, y, z \in[0,1]$ be arbitrarily chosen. A fuzzy implication $I:[0,1]^{2} \rightarrow[0,1]$ satisfies:

(NP) the left neutrality property if $I(1, y)=y$.

(EP) the exchange principle if

$$
I(x, I(y, z))=I(y, I(x, z)) .
$$

(IP) the identity principle if $I(x, x)=1$.

(OP) the ordering property if

$$
x \leq y \Leftrightarrow I(x, y)=1 .
$$

(CP) the contrapositive symmetry with respect to a given fuzzy negation $N$ if $I(x, y)=I(N(y), N(x))$

\subsection{Fuzzy relations and fuzzy preference structures}

Before turning our attention to (fuzzy) preference relations, we recall basic properties of fuzzy relations.
Definition 5 ([13]) Let $X \neq \emptyset$ be a given set. We say that a fuzzy relation $R: X^{2} \rightarrow[0,1]$ is

- reflexive, if $R(x, x)=1$ for all $x \in X$,

- irreflexive, if $R(x, x)=0$ for all $x \in X$,

- symmetric, if $R(x, y)=R(y, x)$ for all $x, y \in$ $X$.

The basic notion in (crisp) preference modelling is preference structure. A preference structure is given by a triple of relations $(P, I, J)$. Since we will proceed to the fuzzy case, in order to avoid confusions instead of relations $P, I, J$, we will consider their characteristic functions using the same notation. This means, given a set of alternatives $\mathbb{A}$, a decision-maker makes one of three possible decisions for each pair $(a, b) \in \mathbb{A}^{2} . P(a, b)=1$ means that $a$ is preferred to $b, I(a, b)=1$ means that $a$ and $b$ are indifferent, and $J(a, b)=1$ means that $a$ and $b$ are incomparable.

We have the following characterisation of crisp preference relations [12]:

(PS1) $I$ is reflexive and symmetric,

(PS2) $P(a, b)+P^{t}(a, b)+I(a, b)+J(a, b)=1$ for all $(a, b) \in \mathbb{A}^{2}$.

Preference structure can be characterised by a large preference relation $R$. Its characteristic function is defined as $R(a, b)=P(a, b)+I(a, b)$. Thus $R$ is a reflexive relation and the whole triple $(P, I, J)$ can be characterised by means of $R$ :

$$
\begin{aligned}
P(a, b) & =R(a, b)(1-R(b, a)), \\
I(a, b) & =R(a, b) R(b, a), \\
J(a, b) & =(1-R(a, b))(1-R(b, a)) .
\end{aligned}
$$

When fuzzifying a preference structure, we use a De Morgan triple $(T, S, N)$. As it was pointed out (e.g., $[4,12])$, the only reasonable construction of a fuzzy preference structure is based on a De Morgan triple $(T, S, N)$ where $T$ is a nilpotent tnorm. Since each nilpotent t-norm is isomorphic to the Łukasiewicz one (see e.g. [8]), we will consider only the Eukasiewicz De Morgan triple $\left(T_{L}, S_{L}, N_{s}\right)$. Then it is possible to construct a fuzzy preference structure (FPS, for brevity) $(P, I, J)$ satisfying the following properties

(FPS1) $I$ is reflexive and symmetric,

(FPS2) $P(a, b)+P^{t}(a, b)+I(a, b)+J(a, b)=1$ for all $(a, b) \in \mathbb{A}^{2}$.

We have already mentioned that, in the crisp case, it is possible to construct a preference structure from the large preference relation. However, in the fuzzy case this way is problematic.

Proposition 1 ([4]) Let $(P, I, J)$ be a FPS, $\mathbb{A}$ be a set of alternatives. Then there is no De Morgan 
triple $(T, S, N)$ such that for all $a, b \in \mathbb{A}$ we have that

$$
\begin{aligned}
R(a, b) & =S(P(a, b), I(a, b)), \\
P(a, b) & =T(R(a, b), N(R(b, a))), \\
I(a, b) & =T(R(a, b), R(b, a)), \\
J(a, b) & =T(N(R(a, b)), N(R((b, a)))) .
\end{aligned}
$$

Because of this negative result, Fodor and Roubens [4] proposed an axiomatic construction of FPS from a large fuzzy preference relation $R$. For the Łukasiewicz De Morgan triple $\left(T_{L}, S_{L}, N_{s}\right)$ we get the following axioms.

1. Independence of Irrelevant Alternatives:

Let $R: \mathbb{A}^{2} \rightarrow[0,1]$ be a large fuzzy preference relation. Then there exist functions $p, i, j:[0,1]^{2} \rightarrow[0,1]$ such that for all $a, b \in \mathbb{A}$ we have

$$
\begin{aligned}
P(a, b) & =p(R(a, b), R(b, a)), \\
I(a, b) & =i(R(a, b), R(b, a)), \\
J(a, b) & =j(R(a, b), R(b, a)) .
\end{aligned}
$$

2. Positive Association Principle:

Function $p$ is increasing in the first and decreasing in the second variable, $i$ is increasing in both variables, $j$ is decreasing in both variables.

3. Symmetry:

Functions $i$ and $j$ are symmetric.

4. $(P, I, J)$ is a FPS for any reflexive relation $R$ : $\mathbb{A}^{2} \rightarrow[0,1]$ such that

$$
S_{L}(P, I)=R, S_{L}(P, J)=1-R^{t} .
$$

Each triple $(p, i, j)$ fulfilling the above four axioms is called a generator triple.

Lemma 1 ([4]) Let $(p, i, j)$ be a generator triple. Then for all $x, y \in[0,1]$ we have that

$$
\begin{array}{lcc}
T_{L}(x, y) \leq & p(x, 1-y) & \leq \min \{x, y\}, \\
T_{L}(x, y) \leq & i(x, y) & \leq \min \{x, y\}, \\
T_{L}(x, y) \leq & j(1-x, 1-y) & \leq \min \{x, y\} .
\end{array}
$$

Moreover, condition (FPS2) and Independence of Irrelevant Alternatives imply

$$
\begin{aligned}
p(x, y)+p(y, x)+i(x, y)+j(x, y) & =1, \\
p(x, y)+i(x, y) & =x .
\end{aligned}
$$

Generator triples are closely related to fuzzy implications. We reformulate Proposition 3.5 from [4] for the Eukasiewicz De Morgan triple

Proposition 2 ([4]) Let $(p, i, j)$ be a monotone generator triple that solves the following system of equations

$$
\begin{aligned}
& S_{L}(p(x, y), i(x, y))=x, \\
& S_{L}(p(x, y), j(x, y))=1-y .
\end{aligned}
$$

Then $I^{\rightarrow}:[0,1]^{2} \rightarrow[0,1]$ defined by

$$
I^{\rightarrow}(x, y)=1-p(x, y),
$$

is a fuzzy implication that fulfils (NP) and the natural negation $N_{I \rightarrow}$ is the standard negation $N_{s}$.

\section{Construction of FPS using quasi-copulas}

We will assume continuity of generator triples $(p, i, j)$.

For each semi-copula $C$ we can introduce two types of flipping.

Definition 6 (see, e.g., [9]) Let $C:[0,1]^{2} \rightarrow$ $[0,1]$ be a semi-copula. A flip in the first variable of $C$ will be denoted by $C_{\text {flip } 1}$ and defined by

$$
C_{\text {flip1 }}(x, y)=y-C(1-x, y) \text {. }
$$

A flip in the second variable of $C$ will be denoted by $C^{\text {flip2 }}$ and defined by

$$
C^{\mathrm{flip} 2}(x, y)=x-C(x, 1-y) .
$$

Keeping the notation from [9] we introduce the survival semi-copula to semi-copulas $C$ such that $T_{L} \leq C \leq T_{M}$

Definition 7 (see, e.g., [9]) Let $C:[0,1]^{2} \rightarrow$ $[0,1]$ be a semi-copula such that $T_{L} \leq C \leq T_{M}$. The survival semi-copula to $C$ will be denoted by $\hat{C}$ and defined by

$$
\hat{C}(x, y)=x+y-1+C(1-x, 1-y) .
$$

J. Kalická showed the following:

Lemma 2 ([7]) Let $C$ be an arbitrary semi-copula. Then $C_{\text {flip1 }}$ is a semi-copula if and only if $C$ is 1-Lipschitz in the second variable, and $C^{\text {flip2 }}$ is a semi-copula if and only if $C$ is 1-Lipschitz in the first variable.

Immediately by Lemma 2 we get that if $C$ is arbitrary semi-copula then $\hat{C}$ is a semi-copula if and only if $C$ is a quasi-copula.

It is possible to model generator triples $(p, i, j)$ using quasi-copulas as follows.

Proposition 3 Let $C_{1}$ be a quasi-copula whose flips are commutative. Then we have that the triple $\left(p_{1}, i_{1}, j_{1}\right)$, given by

$$
\begin{aligned}
p_{1}(x, y) & =C_{1}(x, 1-y), \\
i_{1}(x, y) & =\left(C_{1}\right)^{\text {flip } 2}(x, y)=x-C_{1}(x, 1-y), \\
j_{1}(x, y) & =\left(C_{1}\right)_{\text {flip } 1}(1-x, 1-y) \\
& =1-y-C_{1}(x, 1-y),
\end{aligned}
$$

is a generator triple. 
Proposition 4 Let $C_{2}, C_{3}$ be commutative quasicopulas. Then triples $\left(p_{2}, i_{2}, j_{2}\right)$ and $\left(p_{3}, i_{3}, j_{3}\right)$, given by

$$
\begin{aligned}
p_{2}(x, y) & =\left(C_{2}\right)^{\mathrm{flip} 2}(x, 1-y)=x-C_{2}(x, y), \\
i_{2}(x, y) & =C_{2}(x, y), \\
j_{2}(x, y) & =\hat{C}_{2}(1-x, 1-y)=1-x-y+C_{2}(x, y),
\end{aligned}
$$

and

$$
\begin{aligned}
p_{3}(x, y) & =\left(C_{3}\right)_{\text {flip } 1}(x, 1-y) \\
& =1-y-C_{3}(1-x, 1-y), \\
i_{3}(x, y) & =\hat{C}_{3}(x, y)=x+y-1+C_{3}(1-x, 1-y), \\
j_{3}(x, y) & =C_{3}(1-x, 1-y),
\end{aligned}
$$

respectively, are generator triples.

Generator triples constructed in Propositions 3 and 4 are sketched in Figures 1, 2 and 3 .

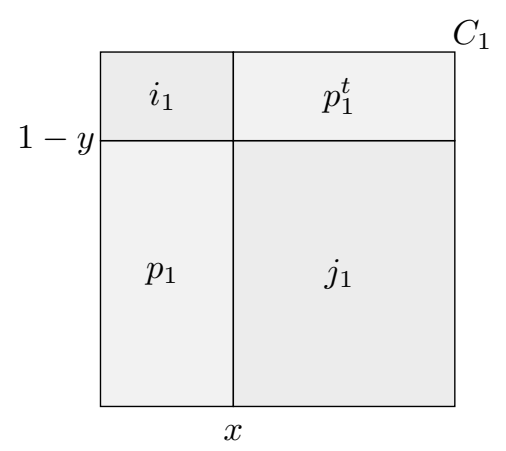

Figure 1: Generator triple $\left(p_{1}, i_{1}, j_{1}\right)$.

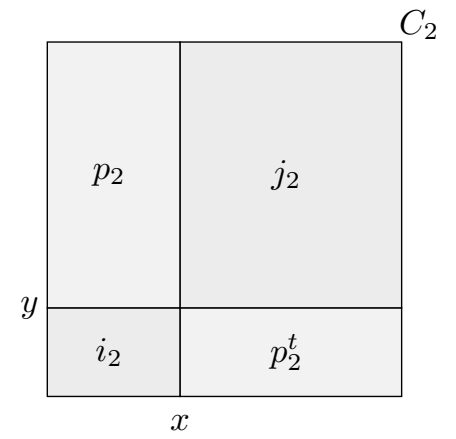

Figure 2: Generator triple $\left(p_{2}, i_{2}, j_{2}\right)$.

For $C_{1}=\left(C_{2}\right)^{\text {flip2 }}, C_{3}=\hat{C}_{2}$ we have that

$$
\left(p_{1}, i_{1}, j_{1}\right)=\left(p_{2}, i_{2}, j_{2}\right)=\left(p_{3}, i_{3}, j_{3}\right) .
$$

This equality further implies the following

$$
\begin{aligned}
p_{1}(x, y) & =C_{1}(x, 1-y)=x-C_{2}(x, y) \\
& =1-y-\hat{C}_{2}(1-x, 1-y) \\
& =1-y-\hat{C}_{2}(1-y, 1-x),
\end{aligned}
$$

where we have used the fact that $C_{2}$ and hence also $\hat{C}_{2}$ are commutative quasi-copulas. If we further

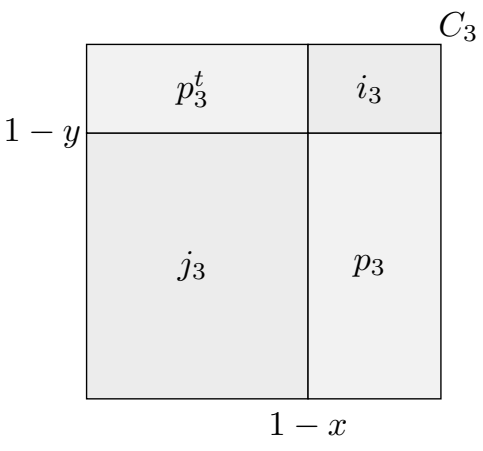

Figure 3: Generator triple $\left(p_{3}, i_{3}, j_{3}\right)$.

assume that $C_{2}=\hat{C}_{2}$, we get

$$
\begin{aligned}
p_{1}(x, y) & =1-y-\hat{C}_{2}(1-y, 1-x) \\
& =1-y-C_{2}(1-y, 1-x) \\
& =C_{1}(1-y, x)=p_{1}(1-y, 1-x) .
\end{aligned}
$$

Proposition 5 Let $C$ be a commutative quasicopula. Then flips

$$
\begin{aligned}
& C^{\text {flip2 }}(x, y)=x-C(x, 1-y), \\
& C_{\text {flip } 1}(x, y)=y-C(1-x, y)
\end{aligned}
$$

are commutative if and only if $C=\hat{C}$.

Propositions 2 and 5 have the following corollary.

Corollary 1 Let $C$ be a commutative quasi-copula. Then fuzzy implication $I^{\rightarrow}:[0,1]^{2} \rightarrow[0,1]$, defined by

$$
I^{\rightarrow}(x, y)=1-x+C(x, y)
$$

fulfils the Contrapositive Symmetry with respect to $N_{s}$ if and only if $C=\hat{C}$.

Remark 2 The fuzzy implication $I^{\rightarrow}$ defined by formula (3) was studied by Grzegorzewski in [5] in case that $C$ is a copula. This fuzzy implication is called the Probabilistic S-Implication. In fact, formula (3) defines an implication if and only if $C$ is a conjunctor which is 1-Lipschitz in the first variable.

Propositions 2 and 5 have yet another corollary for generator triples.

Corollary 2 Let $C$ be a commutative quasi-copula and $(p, i, j)$ be a generator triple fulfilling formulae (1) and (2). Further assume that $i(x, y)=C(x, y)$. Then the following statements are equivalent

- $i(x, y)=j(1-x, 1-y)$,

- $p(x, y)=p(1-y, 1-x)$,

- $C=\hat{C}$.

In general, if $p(x, y)=C(x, 1-y)$ for a quasicopula $C$, then $C$ is not necessarily commutative. Just flips of $C$ have to be commutative.

Proposition 6 Let $C$ be a quasi-copula. Then flips $C^{\text {flip2 }}$ and $C_{\text {flip1 }}$ are commutative if and only if the following holds

$$
C=(\hat{C})^{t}
$$


Summarizing properties of the fuzzy implication $I^{\rightarrow}$ we get the next statement.

Proposition 7 Let $C$ be a continuous conjunctor. Then the following items are equivalent.

- $I^{\rightarrow}$ expressed by the following formula

$$
I^{\rightarrow}(x, y)=1-x+C(x, y)
$$

is a fuzzy implication, yielding (CP) with respect to $N_{s}$ and moreover $I^{\rightarrow}$ has bounds given by the next inequality

$$
I_{S_{M}} \leq I^{\rightarrow} \leq I_{S_{L}}
$$

- $C$ is a quasi-copula such that $C=\hat{C}^{t}$.

Remark 3 We have already mentioned in Proposition 2 (see also [4]) that fuzzy implication $I^{\rightarrow}$ fulfils also (NP) and for the natural negation we have $N_{I \rightarrow}=N_{s}$. These two properties are in fact a corollary to inequality (4).

As it was proved in [5], the probabilistic Simplication (if $C$ is a copula) fulfils (IP) and (OP) if and only if $I^{\rightarrow}$, defined by (3), is the Eukasiewicz implication, i.e., $I^{\rightarrow}=I_{S_{L}}$. In this case we have $C(x, y)=T_{M}(x, y)$.

Straightforwardly we get the following assertion

Lemma 3 Let $C$ be a quasi-copula such that $C=$ $\hat{C}$. Then the fuzzy implication $I^{\rightarrow}$, defined by formula (3), fulfils (IP) and (OP) if and only if $I^{\rightarrow}=$ $I_{S_{L}}$.

Example 1 Let $C:[0,1]^{2} \rightarrow[0,1]$ be the quasicopula defined by

$$
C(x, y)=x^{2} y, \quad \text { if } x+y \leq 1,
$$

and

$$
\begin{aligned}
C(x, y)= & x+y-1+C(1-y, 1-x) \\
= & y(2 x+y-x y-1), \\
& \text { if } x+y>1 .
\end{aligned}
$$

Then $C=(\hat{C})^{t}$. Further we get

$$
\begin{gathered}
C^{\mathrm{flip} 2}(x, y)= \begin{cases}x-x^{2}(1-y), & \text { if } x \leq y, \\
y-y^{2}(1-x), & \text { if } y<x,\end{cases} \\
C_{\text {flip } 1}(x, y)= \begin{cases}2 x y-x^{2} y, & \text { if } x \leq y, \\
2 x y-y^{2} x, & \text { if } x<y .\end{cases}
\end{gathered}
$$

Observe further that

$$
\widehat{C^{\text {flip2 }}}(x, y)= \begin{cases}x-x(1-y)^{2}, & \text { if } x \leq y, \\ y-y(1-x)^{2}, & \text { if } y<x\end{cases}
$$

which means that $C \neq{\widehat{C^{\text {flip2}}}}^{t}$.
The corresponding generator triple (p,i.j) is then given in the following way

$$
\begin{aligned}
p(x, y) & =C(x, 1-y), \\
i(x, y) & =C^{\mathrm{flip} 2}(x, y), \\
j(x, y) & =C_{\text {flip } 1}(1-x, 1-y) .
\end{aligned}
$$

Yet let us check the fuzzy implication $I^{\rightarrow}$ :

$$
\begin{aligned}
I^{\rightarrow}(x, y)= & 1-x+C^{\text {flip2}}(x, y)=1-C(x, 1-y) \\
& =\left\{\begin{array}{c}
1-x^{2}(1-y), \\
\text { if } x \leq y, \\
1-x+y-(1-y)(x-y+x y), \\
\text { if } x>y,
\end{array}\right.
\end{aligned}
$$

and

$$
\begin{aligned}
I^{\rightarrow(1-y, 1-x)=} & y+C^{\text {flip2}}(1-y, 1-x) \\
= & 1-C(1-y, x) \\
= & \left\{\begin{array}{c}
1-(1-y)^{2} x, \\
\text { if } x \leq y, \\
1-x+y-(1-x)^{2} y, \\
\text { if } x>y .
\end{array}\right.
\end{aligned}
$$

This gives that $I^{\rightarrow}$ does not fulfil (CP) with respect to $N_{s}$.

Example 2 Let $C:[0,1]^{2} \rightarrow[0,1]$ be the quasicopula defined by

$$
C(x, y)=x^{2} y^{2}, \quad \text { if } x+y \leq 1,
$$

and

$$
\begin{aligned}
C(x, y)= & x+y-1+C(1-x, 1-y) \\
= & x+y-1+(1-x)^{2}(1-y)^{2}, \\
& \text { if } x+y>1 .
\end{aligned}
$$

Then $C$ is commutative and $C=\hat{C}$. Further we get

$$
\begin{gathered}
C^{\mathrm{flip} 2}(x, y)= \begin{cases}x-x^{2}+2 x^{2} y-x^{2} y^{2}, & \text { if } x \leq y, \\
y-y^{2}+2 x y^{2}-x^{2} y^{2}, & \text { if } y<x,\end{cases} \\
C_{\mathrm{flip} 1}=C^{\mathrm{flip} 2} .
\end{gathered}
$$

For the fuzzy implication $I^{\rightarrow}$ we have that

$$
\begin{aligned}
I^{\rightarrow}(x, y) & =1-x+C(x, y) \\
& = \begin{cases}1-x+x^{2} y^{2}, & \text { if } x+y \leq 1, \\
y+(1-x)^{2}(1-y)^{2}, & \text { if } x+y>1,\end{cases}
\end{aligned}
$$

and

$$
\begin{aligned}
I^{\rightarrow(1-y, 1-x)=} y+C(1-y, 1-x) \\
=\left\{\begin{array}{c}
y+(1-x)^{2}(1-y)^{2}, \\
\text { if } x+y \geq 1, \\
1-x+x^{2} y^{2}, \\
\text { if } x+y<1,
\end{array}\right.
\end{aligned}
$$


i.e., $I^{\rightarrow}$ fulfils $(\mathrm{CP})$ with respect to $N_{s}$. In this case we can define two generator triples:

$$
\begin{aligned}
p_{1}(x, y) & =C(x, 1-y), \\
i_{1}(x, y) & =C^{\mathrm{flip} 2}(x, y), \\
j_{1}(x, y) & =C_{\text {flip } 1}(1-x, 1-y) \\
& =C^{\mathrm{flip} 2}(1-x, 1-y),
\end{aligned}
$$

and

$$
\begin{aligned}
p_{2}(x, y) & =C^{\mathrm{flip} 2}(x, 1-y), \\
i_{2}(x, y) & =C(x, y), \\
j_{2}(x, y) & =C(1-x, 1-y) .
\end{aligned}
$$

\section{Conclusions}

In this paper we have found a necessary and sufficient condition under which continuous generator triples can be constructed via quasi-copulas. In the case that $C$ is a quasi-copula whose flips are commutative, we have shown that this condition is equivalent to the condition that the corresponding probabilistic implication $I^{\rightarrow}$ is continuous, bounded by $I_{S_{M}}$ from below and by $I_{S_{L}}$ from above, and moreover $I^{\rightarrow}$ fulfils $(\mathrm{CP})$ with respect to $N_{s}$.

\section{Acknowledgements}

Martin Kalina has been supported from the Science and Technology Assistance Agency under contract No. APVV-0073-10, and from the VEGA grant agency, grant number 1/0143/11.

This paper is based upon work supported by the Project: Mobility - enhancing research, science and education at the Matej Bel University, ITMS code: 26110230082, under the Operational Program Education cofinanced by the European Social Fund.

\section{References}

[1] M. Baczyński, B. Jayaram, Fuzzy implications, volume 231 Studies in Fuzziness and Soft Computing, Springer, Berlin, 2008.

[2] B. Bassan, F. Spizzichino, Dependence and multivariate aging: the role of level sets of the survival function. In System and Bayesian Reliability, Series of Quality Reliability Engineering Statistics, Vol.5, pages 229-242, World Scientific Publishers, River Edge, NJ, 2001.

[3] F. Durante, E.P. Klement, R. Mesiar, C. Sempi, Conjunctors and their residual implicators: characterizations and construction methods, Mediterranean J.Math. 4:343-356, 2007.

[4] J. Fodor, M. Roubens, Fuzzy preference modelling and multicriteria decision support, Kluwer Academic Publishers, Dordrecht, 1994.

[5] P. Grzegorzewski, Probabilistic implications, Fuzzy sets and Systems, Elsevier, 2013, http://dx.doi.org/10.1016/ j.fss.2013.01.003

[6] P. Hájek, Mathematics of Fuzzy Logic, Kluwer Academic Publishers, Dordrecht, 1998.
[7] J. Kalická, On some construction methods for 1Lipschitz aggregation functions, Fuzzy sets and Systems, 160:726-732, Elsevier, 2009.

[8] E.P. Klement, R. Mesiar, E. Pap. Triangular Norms, Trends in Logic. Studia Logica Library, Vol.8, Kluwer Academic Publishers, Dorderecht, 2000.

[9] R.B. Nelsen, An Introduction to Copulas, second ed., Springer Series in Statistics, Springer, New York, 2006.

[10] V. Novák, I. Perfilieva, J. Močkoř, Mathematical Principles of Fuzzy Logic, Kluwer Academic Publishers,Boston, 1999.

[11] A. Sklar, Fonctions derépartition à $n$ dimensions et leurs marges, Publ. Inst. Statist. Univ. Paris 8: 229-231 1959.

[12] B. Van De Walle, B. De Baets, E. Kerre, A comparative study of completeness conditions in fuzzy preference structures. In proceedings of IFSA'97, vol. III, Academia, Prague, 1997, pages 74-79.

[13] L.A. Zadeh, Similarity relations and fuzzy orderings, Information Sciences, 3:177-200, Elsevier, 1971. 